\title{
Complex damages - Überlegungen zu Asbest aus Sicht eines Rückversicherers ${ }^{1}$
}

\author{
Christian Lahnstein
}

Die aktuellen Asbestszenarien zeigen das gesamte Spektrum der Entwicklungsstadien eines Technologierisikos: boomende Industrien und frühindustrielle Missstände in Entwicklungsländern, in den Industrieländern Generationen kranker Arbeiter als Spätfolge der Nachkriegs-Industrialisierungsphase, aber auch Exzesse des Vorsorgeprinzips beim Asbestausbau.

Der Boom der Asbestindustrie begann vor 100 Jahren. „A big selling point was the ability of asbestos to save lives, both on land and at sea. Asbestos in skyscrapers, offices, theatres, houses, brakes, and ships was already gaining a reputation of saving thousands of lives a year." 2 In einer Übersicht über Theaterbrände der 1870er und 80er Jahre wurde argumentiert, 95\% der dort dokumentierten 2.216 Todesfälle hätten durch Asbest verhindert werden können. ${ }^{3}$ Für einen Moment lebte diese Debatte wieder auf, als nach dem 11. September 2001 die Frage gestellt wurde, ob die Zwillingstürme mit Asbestisolierung etwas länger standgehalten hätten.

Früh begann sich auch der Blick für Gesundheitsrisiken als Kollateralschäden dieser neuen Sicherheitstechnologie zu schärfen. So resümierte der britische Factory Inspector Sir Thomas Legge 1934, „Looking back in the light of present knowledge, it is impossible not to feel that opportunities for discovery and prevention were badly missed. " 4

Die Entschädigung asbestbedingter Krankheiten ist heute für die Versicherungswirtschaft der Prototyp des emerging risk, der komplexeste Fall aller complex damages, der größte und langlebigste aller mass torts. Sicher ist Asbest kein typisches Beispiel eines Massenverfahrens, aber das gilt ebenso für die meisten anderen Fälle - etwa Agent Orange mit seinen militärischen ${ }^{5}$ oder DES mit seinen intergenerationellen Implikationen, ${ }^{6}$ aber auch für den historischen Vorgänger der Asbestprozesse, die eigentlich vergleichbare und dennoch ganz anders verlaufende Geschichte der Entschädigung oder

$1 \mathrm{Zu}$ dem Thema erscheint demnächst eine Publikation der Münchener Rück (Asbestos Worldmap. Anatomy of a Mass Tort), hrsg. von Lahnstein u.a.

2 Tweedale, Magic mineral to killer dust, Turner \& Newall and the asbestos hazard, 2000, 5.

3 Summers, Asbestos and the asbestos industry, 1919, 49.

4 Legge, Asbestosis, Industrial Maladies, London, Oxford University Press, 1934, 190-194.

5 Opfer des Herbizideinsatzes in Vietnam: Vergleich der sieben verklagten Chemieunternehmen mit den US-Veteranen in den 80er Jahren; gegenwärtig in Diskussion: ein Entschädigungsmodell für vietnamesische Opfer.

6 Hierzu zuletzt Wagner, Asbestschäden - Bismarck was right - Entscheidung des englischen House of Lords vom 3. Mai 2006, ZEuP 4/2007, 1130. 
Nicht-Entschädigung der Silikoseopfer in den USA. ${ }^{7}$ Die meisten Massenverfahren sind unwiederholbare historische Abläufe: für die Versicherungspraxis höchst lehrreich, wenn auch für Modellierungszwecke eher ungeeignet.

\section{Drei die Komplexität der Asbestverfahren bewirkende Faktoren}

- Das breite Anwendungsgebiet des Werkstoffs in fast allen Sektoren der verarbeitenden Industrie, in allen Industrieländern und vielen Entwicklungsländern. Die WHO schätzt, dass heute 125 Millionen Menschen beruflichen Asbestrisiken ausgesetzt und jährlich 90.000 Todesfälle darauf zurückzuführen sind. ${ }^{8}$

- Die Zeitdimension: eine das ganze 20. Jahrhundert durchziehende industrielle Nutzung, die keineswegs abgeschlossen ist, denn der im Zeitraum 1980 bis 2000 halbierte weltweite Verbrauch steigt seit der Jahrhundertwende erneut. ${ }^{9}$ Dazu kommen 10- bis 50-jährige Latenzzeiten der Krankheitsbilder; schließlich der Umstand, dass die inzwischen fast 200 Mio Tonnen Asbestfasern, im Lauf von 100 Jahren zutage gefördert, seitdem nicht von der Erdoberfläche verschwunden sind. So ist noch lange mit dem Risiko zu rechnen, vor allem im Bauwesen. 10

- Die Vielfalt der Rechtsgebiete, die - innerhalb jeder nationalen Rechtsordnung mit der Kompensation von Asbestschäden befasst sind: Arbeitsrecht und die verschiedenen Zweige des Sozialrechts, verschiedene Bereiche des Haftungsrechts, vor allem der Arbeitgeber-, Produkt- und Umwelthaftung, Verwaltungsrecht und Staatshaftung, Strafrecht, Insolvenz- und Gesellschaftsrecht. Im Übrigen sind die Regelungen in jedem Land - auch innerhalb der EU - unterschiedlich, und sowohl die Rechtsregeln als auch die Anwendungspraxis verändern sich im Lauf der Jahre.

2. Aus der Kombination dieser drei Faktoren resultierende Hauptprobleme oder Problemcluster der Asbestverfahren

- Intransparenz und schwer überschaubare Interessenkonflikte infolge der Vielzahl beteiligter Parteien: einerseits die Opfer, ihre Familien und Erben sowie Kläger-

7 Vgl. Rosner/ Markowitz, Deadly dust, Silicosis and the on-going struggle to protect workers' health, 2006.

8 World Health Organisation, Elimination of asbestos-related disease, policy paper, September 2006.

9 Vor allem in China, Indien, Thailand und Ukraine.

10 Auch als Risiko nach Naturkatastrophen: nach dem Erdbeben 1995 in Kobe war die Asbestentsorgung der größte Kostenfaktor der Demolierungs- und Aufräumungskosten. Kazan-Allen, Killing the future, asbestos use in Asia, 2007, 24. 
kollektive (Gruppen- und Verbandsklagen) und rückgriffsberechtigte Versicherer, andererseits die beklagten Unternehmen und ihre Rechtsnachfolger, Arbeiterunfallversicherer und Haftpflichtversicherer einschließlich Mit- und Rückversicherern mit wechselnden Beteiligungen im Zeitablauf.

- Die sowohl haftungsrechtlichen als auch prozess- und versicherungsrechtlichen Fragen zeitlicher Zuordnung und Verjährung sowie der Aggregierung von Einzelschäden. Damit zusammenhängend die ungelösten Probleme der Sicherung künftiger Ansprüche und des Umgangs mit der immer häufigeren Situation, dass Expositionen aus der Vergangenheit bekannt, aber deren künftige Krankheitsfolgen unbekannt sind.

- Kausalitätsnachweis: Fragen der general causation beim Zusammenwirken von Asbest mit anderen Krankheiten und Schadstoffen, früher mit Tuberkulose und Silikose sowie anderen Industrieemissionen, dann das Zusammenwirken mit Tabak und Verkehrsemissionen bei Lungenkrebs. Specific causation: hier stellen die unterschiedlichen Rechtsgebiete unterschiedliche Anforderungen. Die britische Haftungsrechtler und Haftpflichtversicherer haben sich im Rahmen der Arbeitgeberhaftung in den letzten Jahren auf die Frage der gesamtschuldnerischen Haftung mehrerer sukzessiver Arbeitgeber desselben Arbeitnehmers konzentriert. ${ }^{11}$ In den Niederlanden hat sich, ebenfalls im Rahmen der Arbeitgeberhaftung, der oberste Gerichtshof in einem Lungenkrebsfall für eine erstaunlich weitgehende Proportionalhaftung ausgesprochen. ${ }^{12}$ Danach haftet ein Arbeitgeber nur entsprechend seinem Verursachungsanteil unter Berücksichtigung aller anderweitigen Verursachungsanteile wie Rauchen, genetische Konstitution und das Hintergrundrisiko allgemeiner Luftverschmutzung. In den USA steht im Rahmen der Produkthaftung die Frage im Vordergrund, wie einzelne Zulieferer der Arbeitgeber haften. Dabei scheinen gegenwärtig die Gerichte nach zwischenzeitlich fast unglaublicher Lockerung und massivem Missbrauch zu den restriktiven Kriterien der 80er Jahre zurückzukehren. Das Pendel schlägt zurück. Autohersteller versuchen jetzt, die Gerichte zu überzeugen, dass Asbest in Bremsklötzen kein Risiko ist und die umfangreiche medizinische Literatur, die dies nahelegt, ,junk science". ${ }^{13}$ In der Arbeiterunfallversicherung muss der Nachweis der Berufsbezogenheit der Krankheit erbracht werden. Hier haben die Arbeiterunfallversicherer unterschiedliche Kriterien und Praktiken entwickelt. Die Sozialisierung der Kosten von Langzeitrisiken in wie auch immer gruppierte Unternehmensklassen erübrigt viele Probleme individueller Zurechnung. Im Übrigen dürfte es im Rah-

11 Vgl. Wagner (Fn. 6) 1122-1136.

12 Hoge Raad 31. März 2006, Überlegungen zum Urteil in: Faure/ Hartlief, Länderbericht Niederlande, in: Koziol/ Steininger (eds.), European Tort Law 2006, 2007, 347-350.

13 Chapin v. DaimlerChrysler et al, case No. 133178, Supreme Court of Michigan; hierzu Welch, Asbestos exposure causes mesothelioma, but not this asbestos exposure, International Journal of Occupational and Environmental Health vol.13/3 Jul/Sep 2007, 318-327. 
men eines Arbeiterunfallversicherungssystems eher gelingen, einheitliche Anerkennungs- und Zurechnungskriterien bei der Entschädigung von Berufskrankheiten zu entwickeln, als es dem Haftungsrecht möglich zu sein scheint. ${ }^{14}$

- Die unklare Koordination der unterschiedlichen mit der Entschädigung von Asbestopfern befassten Rechtsgebiete und Zuständigkeiten innerhalb einer Rechtsordnung. Dieser Aspekt kommt in den auf das Haftungsrecht konzentrierten Asbestdebatten in UK und den USA zu kurz. Die so überaus umfangreichen USAnalysen sind unvollständig, was die Involvierung der Personen- und Sozialversicherer betrifft. Da internationale Asbestkonferenzen der Versicherungswirtschaft ihren Schwerpunkt in der Regel auf diese Länder setzen ${ }^{15}$, bleibt der Blick auf die komplexen Strukturen der anderen Märkte sowie das individuelle Profil jedes einzelnen Marktes deshalb oft verstellt.

\section{Unterschiedliche Entschädigungssysteme}

In den Niederlanden entschied man 1967, die Entschädigung berufsbedingter Unfälle und Krankheiten in die unspezifischen Sozialversicherungszweige der Invaliditäts- und Krankenversicherung zu integrieren und damit die oft problematische Differenzierung zwischen beruflichen und außerberuflichen Ursachen zu erübrigen - same injury, same need, same benefit. In den meisten Ländern blieb es bei der Zuständigkeit der Arbeiterunfallversicherungen, mit allerdings höchst unterschiedlichen Anerkennungspraktiken. In einigen Ländern wie Japan und Spanien sind auch die freiwillige kollektiven Zusatzversicherungen von Bedeutung.

Was die Rolle des Haftungsrechts betrifft, so sind Produkt-, Umwelt- und Arbeitgeberhaftung zu unterscheiden:

- Die Produkthaftung steht seit 35 Jahren in den USA im Vordergrund - bislang nur dort, obwohl die Haftungsregeln weltweit einigermaßen einheitlich sind.

- Die Umweltschäden wegen Staubbelastung von Nachbarn asbestverarbeitender Betriebe, Familienangehörigen von Arbeitnehmern und sonstiger Dritter sind vor allem in dicht besiedelten Industrieländern wie Japan, den Niederlanden und Belgien beachtlich, in Entwicklungsländern mit uneinheitlichen Umweltstandards wie Südafrika, Indien oder China, und in Ländern mit eigener Primärproduktion wie Australien.

- Mit den wichtigen Ausnahmen von Nordamerika (Kanada, USA, Mexiko) und einigen zentraleuropäischen Ländern (Belgien, Deutschland, Österreich) steht in fast allen Ländern die Arbeitgeberhaftung im Vordergrund. Die sozialrechtlichen

14 In diesem Sinn auch Wagner (Fn. 6), 1136.

15 Anders die internationalen Konferenzen der Arbeitsbehörden, Sozial- und Arbeiterunfallversicherer, in denen die Privatversicherung allerdings kaum vertreten ist, so wenig wie in denen der Opferverbände und der global agierenden Asbestos ban-Bewegung. 
Rahmenbedingungen definieren die Arbeitgeberhaftung in jedem Land auf unterschiedliche Weise, sowohl was die Direktansprüche der Arbeitnehmer als auch was den Rückgriff der Arbeiterunfallversicherer oder Dritter betrifft. So vielgestaltig wie die Haftungsvarianten sind die Varianten der Arbeitgeberhaftpflichtversicherung: stand alone-Policen wie in UK, Irland oder Israel, stillschweigender oder ausdrücklicher Einschluss in der Betriebshaftpflichtversicherung wie in der Mehrzahl der Länder (mit Varianten im Deckungsumfang und in der Versicherungsdichte), oder eine Kombination mit der Arbeiterunfallversicherung, ebenfalls mit Varianten: kumulativ wie in Hongkong, alternativ wie in Indien oder Singapur, oder nur punktuell ergänzend wie in den USA; der australische Versicherungsmarkt hat im Lauf der Jahre alle drei Varianten durchexerziert. Im Übrigen stehen die Versicherungslösungen in keiner festen Beziehung zu den Haftungsvarianten, vielmehr finden sich fast alle denkbaren Kombinationen.

- Die sich über lange Zeiträume erstreckende Kompensation asbestbedingter Gesundheitsschäden verteilt sich damit auf unterschiedliche Versicherungskollektive. Initiativen von Opferverbänden führen zunehmend zu staatlich organisierten Entschädigungsfonds wie in Frankreich und zuletzt in Belgien, Slowenien und Japan. All diese Fonds werden in erster Linie durch die Arbeiterunfallversicherer und damit indirekt durch die Arbeitgeber finanziert, ergänzend durch staatliche Beteiligung im Hinblick auf die nicht arbeitsplatzbezogenen Umweltrisiken, auf alte Verbindlichkeiten der nicht mehr existierenden Unternehmen und der Staatsunternehmen, letztlich auch im Hinblick auf eine generelle staatliche Verantwortung als Riskmanager und Regulator. Die Haftungsfragen werden damit tendenziell zu Fragen der Rückgriffsrechte und -praktiken dieser Fonds. Eigenartig - aber nicht unter diesem Aspekt in Frage gestellt - erscheint in diesem Zusammenhang die Struktur des inzwischen aus anderen Gründen gescheiterten USFondmodells, des Fair Act von 2005. Hier waren weder die Arbeiterunfallversicherer noch die Bundesregierung als Financiers vorgesehen, sondern das heterogene Kollektiv der aktuell verklagten Unternehmen und deren Versicherer: ein erneuter Versuch eines global settlement, seitens des Gesetzgebers nun ebenso gescheitert wie in den 90er Jahren seitens der Gerichte. ${ }^{16}$

\section{US-Lektionen}

Eine gängige Erkenntnis aus der US-Asbestgeschichte ist, dass das Risiko in der Haftpflichtversicherung zu spät ausgeschlossen wurde. Betrachtet man allerdings die früheren Phasen dieser Geschichte, so erscheint als eigentlicher Fehler eher, das Risiko nicht von Anfang an adäquat versichert zu haben.

$16 \mathrm{Zu}$ den gescheiterten class actions Hensler, Asbestos Litigation in the United States, Triumph and Failure of the Civil Justice System, Connecticut Insurance Law Journal, 2006, 266-269. 
- Zu Beginn des 20. Jahrhunderts war industrial insurance, eine vereinfachte Form der Lebensversicherung, der wichtigste Privatversicherungszweig der Arbeiterklasse. 1910 waren 23 Mio Personen versichert, ein drei Milliarden DollarGeschäft, was ungefähr der heutigen wirtschaftlichen Bedeutung der Workers' compensation-Versicherung entspricht (ca. 60 Mrd. USD). 80\% dieses Geschäfts teilten sich zwei Gesellschaften, Prudential Life und Metropolitan Life. Die Chefstatistiker dieser Gesellschaften, Frederick Hoffman und Louis Dublin, waren Pioniere in der Erforschung der Krankheits-, Invaliditäts- und Todesraten und -ursachen in den einzelnen Bevölkerungsgruppen und speziell von Industriearbeitern. ${ }^{17}$ Bereits 1918 konstatierte Frederick Hoffman die Weigerung der Lebensversicherer, asbestexponierte Arbeiter zu versichern.

- Die zweite Gelegenheit, die Entschädigungsfrage anzugehen, wurde in den 30er Jahren verpasst. Hier gelang es Industrie und Versicherern gemeinsam, eine Common law-Arbeitgeberhaftungs-Klagewelle zu stoppen, und zwar durch Abschaffung der employers' liability : tort reform und Immunität der Arbeitgeber durch Erstreckung der Arbeiterunfallversicherung auf Berufskrankheiten, ähnlich wie 20 Jahre früher im Zusammenhang mit Arbeitsunfällen in den USA und wie 1884 in Deutschland. Anders als in Deutschland, erwiesen sich die langfristigen Folgen dieser tort reform als fragwürdig. Vergleicht man die gegenwärtigen Haftungsprozesse in den USA und in Großbritannien, so zeigt sich, dass die UKLösung über Arbeitgeberhaftung, bei aller auch heute noch nicht überschaubaren Problematik, der US-Lösung über Hersteller- und sonstige Dritthaftungen wohl immer noch vorzuziehen ist. Sie ist transparenter, das Kollektiv der Arbeitgeber ist weniger heterogen, und das selbständig finanzierte employers'-liabilityGeschäft kann auch steigende Kosten umlegen oder sollte es jedenfalls tun. Denn die employers'-liability-Versicherer könnten hier durchaus mehr das Argument benutzen, dass die britischen Arbeitgeber anders als die kontinentaleuropäischen oder US-amerikanischen nicht die Kosten der Arbeiterunfallversicherung tragen, da sowohl die Invaliditätsrenten als auch die Krankenkosten - industrial injuries disablement benefits und National Health Service - durch Steuern finanziert werden. Die Gesamtkosten der Arbeitgeber sind deswegen in UK vergleichsweise niedrig.

- Die dritte Gelegenheit - workers' compensation - ergab sich ab den 40er Jahren. Auch sie wurde nicht oder nur sehr zögerlich wahrgenommen (aber auch die europäischen Arbeiterunfallversicherer haben lange gebraucht, bis sie begannen, sich des Problems anzunehmen). Ein großer Teil der Asbestfälle wurde nicht entschädigt infolge von Verjährungsregeln, Karenzzeiten und zu hohen Anforderungen an den Kausalitätsnachweis. Die Leistungen waren niedrig. Die Versicherer neigten dazu, Ansprüche abzulehnen, um das Prämienniveau wettbewerbsfähig zu halten. Die Arbeitgeber desgleichen, um keine Prämienzuschläge zu riskieren. Selbst die Arbeitnehmer waren an Krankheitsdiagnosen nicht interessiert, um keine Kündigung zu riskieren. Eine im internationalen Vergleich unübliche, aber bis heute nicht in Frage gestellte Subsidiaritätsregel führte dazu, dass auf eine In-

17 Rosner/ Markowitz (Fn. 7), 24-28. 
anspruchnahme der Arbeiterunfallversicherung ganz verzichtet wurde, als in den 70er Jahren die Haftung Dritter ins Blickfeld rückte. Nach dieser Regel kann der Arbeiterunfallversicherer seine Leistungen vom Arbeitnehmer zurückfordern, wenn der Arbeitnehmer von dritter Seite entschädigt wird. 18

- Die Entschädigung der Asbestfälle ist weltweit unbestritten eine Hauptaufgabe der Arbeiterunfallversicherung. In vielen Industrieländern sind gegenwärtig etwa die Hälfte aller Todesfälle durch Berufskrankheiten und Arbeitsunfälle durch Asbest bedingt, wobei die Asbestfälle zunehmen und die sonstigen tödlichen Berufskrankheiten und die Arbeitsunfälle abnehmen. ${ }^{19}$ Dies gilt auch für die USA. ${ }^{20}$ Der Kostenaufwand für Asbestfälle liegt im Verhältnis zum Gesamtaufwand der Arbeiterunfallversicherung für Arbeitsunfälle und Berufskrankheiten bei ca. 5\%. ${ }^{21}$ In den USA liegt er nach offiziellen Angaben ,unter 1\%“, nach unserer Einschätzung weit darunter. Ein Aufwand von 5\% sollte für diesen Versicherungszweig alles in allem kein unlösbares Problem darstellen. In den USA entspräche das gegenwärtig 2.5 Mrd. \$US jährlich.

\section{Wahrscheinlichkeit haftungsrechtlicher Massenverfahren}

Die in den meisten Ländern denkbaren Massenklageszenarien der Arbeitgeberhaftung wurden vor 15 Jahren in einer Publikation der Münchener Rück verdeutlicht. ${ }^{22}$ In der Tat gewann die Arbeitgeberhaftung in Asbestfällen an Gewicht, besonders deutlich in Frankreich, wo sie inzwischen durch Fondslösungen überlagert wurde. In anderen Ländern, etwa Italien oder Spanien, hat sie sich bislang eher weniger als erwartet realisiert. Dafür gibt es unterschiedliche Gründe.

Der Stellenwert des Themas auf den aktuellen politischen Agenden ist zweifelhaft. War er hoch wie zeitweise in Deutschland (in den 80er Jahren) oder in Frankreich (in den 90er Jahren), so auch deswegen, weil der Nebenaspekt des Asbest-Umweltrisikos überschätzt wurde, mit dem Ergebnis oft exzessiver Asbestsanierungsaktivitäten, etwa

18 Hierzu Hanlon, An elegy for the Fair Act, Connecticut Insurance Law Journal, 2006, 536, 546.

19 In Deutschland 2005 laut DGUV-Statistiken 1540 asbestbedingte Todesfälle gegenüber 944 Todesfällen durch sonstige Berufskrankheiten und 589 tödlichen Arbeitsunfällen (ohne Wegeunfälle); damit Anteil der Asbestfälle erstmals über 50\%.

20 In den meisten Entwicklungsländern - China, Indien, Lateinamerika - liegt der relative Anteil von Asbestkrankheiten deswegen niedriger, weil sowohl die Zahl tödlicher Arbeitsunfälle als auch die Zahl anderer tödlicher Berufskrankheiten wie Silikose oder Bleivergiftung wesentlich höher ist. $\mathrm{Zu}$ den Schwierigkeiten der Arbeiterunfallversicherer, vor diesem Hintergrund Latenzrisiken in ihren Leistungskatalog aufzunehmen: Zimmer, Berufskrankheiten, eine globale Herausforderung, Die BG 11/2007, 432-437.

21 In Ländern mit bereits hohem Entschädigungsniveau in Asbestfällen; in Deutschland beträgt der jährliche Entschädigungsaufwand zur Zeit jährlich zwischen 300 und 350 Mio $€$ bei 6 bis 7 Mrd $€$ Entschädigungsaufwand für alle Arbeitsunfälle und Berufskrankheiten.

22 Münchener Rück, Die Haftung des Arbeitgebers, 1993. 
in Schulgebäuden. ${ }^{23}$ Das Kernproblem, die Gesundheitsrisiken der Industriearbeiter, interessierte die Öffentlichkeit weniger, im Übrigen stehen andere Fragen der Sozialpolitik im Vordergrund und in Entwicklungsländern auch andere Gesundheitsrisiken. Zur sozialen Unsichtbarkeit des Problems trägt bei, dass in der Regel ältere Arbeiter betroffen sind, oft Raucher, so dass Asbest als Mitursache auch in den Industrieländern übersehen wird; dass die Vorgänge geografisch gestreut sind und eine Vielzahl von Verursachern in Betracht kommen; und dass unterschiedliche, oft wenig koordinierte Rechtsgebiete und Verfahrenswege involviert sind, die aber in den sozialstaatlich orientierten Industrieländern eine Grundversorgung sicherstellen.

Andererseits wird auch in den alten Industrieländern infolge der langen Latenzzeiten die Zahl der Neuerkrankungen aus Exponierungen vergangener Jahrzehnte in den nächsten Jahren noch zunehmen. Diese Neuerkrankungen werden bei verbessertem medizinischem Informationsstand und längerer Lebenserwartung schärfer wahrgenommen. Entsprechendes gilt für den juristischen Informationsstand der Beteiligten und die Durchsetzung bestehender Rechtsansprüche einschließlich der wachsenden Bedeutung von Verbandsklagen.

In den Entwicklungsländern versuchen die global vernetzten Opferverbände den Themen Asbestverbot und Opferentschädigung politisches Gewicht zu geben, unterstützt von ILO und WHO. ${ }^{24}$ Empfohlen wird koordinierte Aktion in den Regionen oder weltweit, da erfahrungsgemäß unilaterale Verbote zur Verlagerung des Risikos in Länder mit schlechter reguliertem Arbeits- und Umweltschutz führen. So im Fall von Asbesttextilien und Bremsklötzen, deren Herstellung von Japan nach Korea, dann in den 90er Jahren von Korea nach China und in andere südostasiatische Länder verlagert wurde.

Der größte Teil der exponierten Arbeiter findet sich in der weitgehend unregulierten informellen Bauindustrie einschließlich der über 100.000 Arbeiter, die in Indien und Bangladesh mit dem Abwracken von Schiffen beschäftigt sind. Angesichts dieser Rahmenbedingungen sind die Argumente der Bergbau- und Asbestzementindustrie in Kanada, Brasilien, Thailand, Indien und den Ländern der ehemaligen Sowjetunion zu controlled use wenig überzeugend, ${ }^{25}$ wenn auch bislang durchaus erfolgreich. Hier sollte die Versicherungswirtschaft drei Ziele unterstützen: weltweites Asbestverbot, Deckung durch Arbeiterunfallversicherung und Fonds sowie selektive Haftpflichtversicherung im Bauwesen, also Deckung nur für Unternehmen, die ausreichende Standards garantiere.

23 Vgl. Franßen, Einleitung zu Krebsrisiko und Luftverunreinigung, Dokument zur 16. wissenschaftlichen Fachtagung der Gesellschaft für Umweltrecht, 1992; auch in den USA drängten Eltern auf Asbestsanierung von Schulen, schlossen sich aber den eher zurückhaltenden Expertenmeinungen an, sobald die konkreten ökonomischen und organisatorischen Folgen zutage traten, vgl. Sunstein, Gesetze der Angst, jenseits des Vorsorgeprinzips, 2005, 74.

24 S. Fn. 7.

25 Z.B. des Canadian Chrysotile Institute oder der Chrysotile International Alliance of Trade Unions. 


\title{
Einschränkung des Versicherungsumfangs in der deutschen gesetzlichen Unfallversicherung im Hinblick auf Wegeunfälle und Berufskrankheiten
}

\author{
Prof. Dr. Maximilian Fuchs
}

\section{Vorbemerkung}

Wegeunfälle und Berufskrankheiten stellen dar, was man im Versicherungswesen als Versicherungsfälle bezeichnet. Der Versicherungsfall ist die zentrale Kategorie jeder Versicherung ${ }^{1}$. Versicherung hat es mit der Bewältigung von Risiken und ihrer Verwirklichung zu tun. Der Versicherungsfall ist die Verwirklichung des versicherten Risikos. Und das Vorliegen des Versicherungsfalls löst die Leistungen aus. Vor diesem Hintergrund leuchtet es ein, dass die rechtliche Fixierung des Versicherungsfalls von elementarer Bedeutung für die Funktionsweise des betreffenden Versicherungszweiges ist.

In diesem Sinne möchte ich auch mein Referat angelegt wissen. Im Vordergrund steht die positiv-rechtliche Darstellung der beiden Versicherungsfälle. Ebenso notwendig ist aber gleichzeitig die Frage nach der Rationalität der zu besprechenden Regelungen. Diese Rationalität kann nur bewertet werden, wenn wir die beiden Versicherungsfälle im Rahmen des gesamten Systems betrachten, das die gesetzliche Unfallversicherung bildet. Die Bewältigung von Körperschäden ${ }^{2}$ wird ja auch von anderen Zweigen der Sozialversicherung geleistet, etwa der Krankenversicherung und Rentenversicherung. Was die Unfallversicherung zu einem speziellen Entschädigungssystem macht, liegt in der Besonderheit begründet ${ }^{3}$, dass

- $\quad$ aus dem großen Feld eintretender Körperschäden diejenigen herausgefiltert werden, die sich kausal auf eine versicherte Tätigkeit zurückführen lassen und

- die Verantwortung für die Kompensation dieser Schäden ausschließlich den Unternehmen überantwortet ist.

Nach der Konzeption des Gesetzes besteht also durchaus eine Verantwortlichkeit der Unternehmen im Sinne der bürgerlichen Haftungsregelungen. Diese Haftungsverant-

1 Zur zentralen Rolle des Versicherungsfalls Weyers, Versicherungsvertragsrecht, 2. Aufl. 1995 Rn. 34 und 551.

2 Zum System der Bewältigung von Körperschäden im deutschen Recht s. Fuchs, Zivilrecht und Sozialrecht, 1992, S. $168 \mathrm{ff}$.

$3 \mathrm{Zu}$ den Grundprinzipien und -mechanismen der gesetzlichen Unfallversicherung s. Gitter, Schadensausgleich im Arbeitsunfallrecht, 1969; Fuchs, Die Legitimation der gesetzlichen Unfallversicherung, SGB 1995, 421 ff.; ders., Struktur und Legitimation der gesetzlichen Unfallversicherung: Eine rechtsvergleichende Betrachtung, in: Internationale Revue für Soziale Sicherheit, 1997, 19 ff. 